\title{
Detection limits of significant prostate cancer using multiparametric MR and digital rectal examination in men with low serum PSA: Up-date of the Italian Society of Integrated Diagnostic in Urology
}

\author{
Andrea B. Galosi ${ }^{1}$, Erika Palagonia ${ }^{1}$, Simone Scarcella ${ }^{1}$, Alessia Cimadamore ${ }^{2}$, Vito Lacetera ${ }^{3}$, \\ Rocco F. Delle Fave ${ }^{1}$, Angelo Antezza ${ }^{1}$, Lucio Dell'Atti ${ }^{1}$ \\ ${ }^{1}$ Division of Urology, School of Medicine, Università Politecnica delle Marche, Ancona, Italy; \\ ${ }^{2}$ Division of Pathology, School of Medicine, Università Politecnica delle Marche, Ancona, Italy; \\ ${ }^{3}$ Division of Urology, Azienda Ospedaliera Marche Nord, Pesaro, Italy.
}

\begin{abstract}
Summary Reasons why significant prostate cancer is still missed in early stage were investigated at the 22nd National SIEUN (Italian Society of integrated diagnostic in Urology, Andrology, Nephrology) congress took place from 30th November to 1st December 2020, in virtual modality. Even if multiparametric magnetic resonance (MR) has been introduced in the clinical practice several, limitations are emerging in patient with regular digital rectal examination (DRE) and serum prostate specific antigen (PSA) levels approaching the normal limits. The present paper summarizes highlights observed in those cases where significant prostate cancer may be missed by PSA or imaging and DRE. The issue of multidisciplinary interest had been subdivided and deepened under four main topics: biochemical, clinical, pathological and radiological point of view with a focus on PI-RADS 3 lesions.
\end{abstract}

KEY WORDS: Prostate cancer; Prostate needle biopsy; Radical prostatectomy; Diagnosis; Magnetic Resonance Imaging.

Submitted 9 January 2021; Accepted 21 January 2021

\begin{abstract}
INTRODUCTION
The present paper summarizes highlights in the field of prostate cancer ( $\mathrm{PCa}$ ) focusing on significant types that do not rise serum PSA, those that are undetected by imaging or those that are unpalpable. This issue rises several implications such as development of early PCa and limitations in cancer detection using updated technologies $(1,2)$. The knowledge is useful for patient and physician information before any further invasive diagnostic procedure such as biopsy or before any therapy of concurrent benign prostatic hyperplasie (BPH) (3). The believes of most men is that every cancer is detected if we use magnetic resonance given that prostatic specific antigen (PSA) and digital rectal examination (DRE) may miss sometimes early cancer, therefore prostate mpMR (magnetic resonance) imaging is extremely required even if is not clinically indicated. As examples of this we refer to two clinical scenarios commonly observed in clinical practice: the first, is Tlb PCa detected after BPH surgery in patients with in-range PSA and normal DRE, the second, the inci-
\end{abstract}

dence of those significant PCa undetected in screening trials that are even now responsible of specific mortality and are called "escapes cancers". The occurrence of one of the above scenarios is overwhelming for the patients and unpredicted for the physician. Then we addressed limitations and reasons to understand why early significant cancer may be still missed nowadays.

These issues of multidisciplinary interest had been subdivided and deepened under four main arguments: the radiological point of view, biochemical and clinical point of view, pathological point of view and focus on PI-RADS 3 lesions: transition zone (TZ) vs peripheral zone (PZ).

\section{Biochemical and clinical point of view}

Evaluate the presence of $\mathrm{PCa}$ in case of men with low PSA level is a new challenge to reduce the mortality due to PCa and increase the detection in case of disease risk. The National comprehensive cancer network (NCCN) (version 2020.1) described the low level of PSA as the presence of a serum PSA $<3 \mathrm{ng} / \mathrm{ml}$ according to Beckman standard, which cut-off is $4.0 \mathrm{ng} / \mathrm{ml}$. This value translated to $\mathrm{WHO}$ is $3.0 \mathrm{ng} / \mathrm{ml}$. Over time the international guidelines changed dramatically based on results of milestone studies by Thompson et al. (4). They presented a clinical trial in which 9459 men were analysed for a PCa Prevention Trial (PCPT): 2950 men presented a PSA level $<4.0 \mathrm{ng} / \mathrm{ml}$ and negative DRE. All the population received biopsy at the end study per protocol.

The prevalence of $\mathrm{PCa}$ was $15.2 \%$ in the group with low PSA level and the incidence of csPCa is summarized in Table 1 . That means that there is a csPCa also in patients with low PSA level. To limit the number of patients to undergo prostate biopsy other clinical evaluations need to be considered: DRE, imaging, prostate volume, age and genetic risk. Advantage must be balanced to probability of prostate biopsy complications (5).

Digital rectal examination and PSA

The decision to do a prostate biopsy can change after the DRE. Based on this fact, Halpern et al. analysed the DRE of 35,350 men and stratified the results by PSA level and 
Table 1.

Correlation of PSA level, prostate cancer (PCa) and high grade PCa (Gleason score $\geq 7$ ) (from Thompson et al. 2004).

\begin{tabular}{|lrrrrr|}
\hline PSA level $\mathrm{n} / \mathrm{ml}$ & $3.1-4$ & $2.1-3.0$ & $1.1-2.0$ & $0.6-1.0$ & $<0.5$ \\
\hline Men with PCa $(\mathrm{n}=449)$ & $26.9 \%$ & $23.9 \%$ & $17.0 \%$ & $10.1 \%$ & $6.6 \%$ \\
\hline Men with high grade PCa (Gleason Score $\geq 7)(\mathrm{n}=67)$ & $25 \%$ & $19.1 \%$ & $11.8 \%$ & $10 \%$ & $12.5 \%$
\end{tabular}

DRE positivity (6). Overall, the incidence of clinically significant PCa was higher in men with suspicious DRE independently of the PSA level. Furthermore, the results highlight that in patients with PSA level between 3-4.9 $\mathrm{ng} / \mathrm{ml}$ the positivity of DRE increased the risk of clinically significant $\mathrm{PCa}$ from $9.2 \%$ to $15.7 \%$; in patients with a level between 2-2.9 from 3.5\% to 6.5\%; between 1-1.9 $\mathrm{ng} / \mathrm{ml}$ from $1.2 \%$ to $2.3 \%$ and in patients with a PSA $<1$ $\mathrm{ng} / \mathrm{ml}$ from $0.2 \%$ to $0.7 \%$. Positive DRE is correlated with cancer even at low level of PSA.

\section{Age and PSA}

Another important aspect is the age of this men and how the time could influence the probability of develop PCa. In particular, a study analysed the probability of men to develop a PCa after 25 years from the first assessment (7). In the range from 37 to 42 years of age with a low PSA level the risk to have a PCa metastasis after 25 years increased with concentrations in the highest $10^{\text {th }}$ of PSA $(\geq 1.3 \mathrm{ng} / \mathrm{ml})$ and the highest quarter of PSA $(\geq 0.9$ $\mathrm{ng} / \mathrm{ml})$. In the range of 45 to 49 years of age the risk to develop cancer metastasis or to die for PCa increased in patients with a PSA level in the highest decile $(\geq 1.6$ $\mathrm{ng} / \mathrm{ml}$ ) and the highest quarter of PSA $\geq 1.1 \mathrm{ng} / \mathrm{ml}$.

\section{PSA velocity}

Lifelong PSA trend can help for rigorous follow-up of young men. This evaluation is called PSA Velocity (PSAV) and calculates how quickly PSA rises over time. The increase in PSA is greater in patients who have a higher risk of developing a lethal PCa (8). There is a cut-off level of $0.35 \mathrm{ng} / \mathrm{ml}$ per year of PSAV which could individualize patients with the risk of death from PCa. Men having a PSAV above 0.355 tol0 years before the diagnosis of the tumor, had a relative risk of PCa death of 10.7 compared to men with a lower PSAV. These results were confirmed extending the retrospective analysis to 15 years, with a relative risk of 4.7 .

Moreover, Vickers et al. assessed that only $8 \%$ of men with a PSA $<2.5 \mathrm{ng} / \mathrm{ml}$ and negative DRE had a PSAV over $0.35 \mathrm{ng} / \mathrm{ml} /$ year and that in this population the risk to develop a PCa was $18 \%$ while in a population with a lower PSAV the risk was 14\% (9). However, the risk of csPCa is not high in both the two groups. These considerations can help us to better evaluate and follow certain young patients with normal PSA and negative DRE.

\section{PSA density and $m p M R$}

PSA Densisty (PSAD) is obtained dividing serum total PSA value by prostate volume calculated by imaging (transrectal ultrasound or mpMR) (10). Since 1990, the PSAD enhanced the specificity of cancer detection in men with a normal DRE and an intermediate PSA level (11). Recently, several studies demonstrated that the combination of PSAD and mpMRI findings allows the better risk stratification in a cohort of biopsy-naive and previously negative biopsy patients. Pagniez et al. increased the negative predictive value of mpMRI from $84.4 \%$ to $90.4 \%$ by using the PSAD with a cut off of $0.15 \mathrm{ng} / \mathrm{ml} / \mathrm{cc}$ (12).

Even in cancer active surveillance an emerging body of evidence is growing. Roscigno et al. evaluated the role of PSAD in identifying patients presenting a different risk of reclassification at confirmatory or follow-up biopsy: higher PSAD was associated with higher risk of reclassification for all PI-RADS lesions (13). PSAD was an independent predictor of reclassification using 0.20 as cut-off.

Furthermore, Bhat et al. showed that none of the patients in active surveillance presented a risk of disease reclassification in case of PI-RADS scores of 1 to 3 and PSAD 0.15 $\mathrm{ng} / \mathrm{mL} / \mathrm{cc}$ as upper limit (14). PSAD might spare some men from the morbidity associated with a prostate biopsy and diagnosis of low-grade prostate cancer, therefore should be included in the flow-chart of prostate cancer diagnosis.

\section{Low or normal PSA and mpMR}

The negative predictive value (NPV) of mpMR is variable: in literature there are many studies that report a NPV more than $80 \%$ and others that set this value around $60 \%$ (15). The main variable to set NPV is the type of population taken into account for the analysis. If we considered a population with a low risk of PCa the NVP is $50 \%$, this means that a negative mpMRI in a patient halves the risk of $\mathrm{PCa}$, but a very high residual risk remains. On the other hand, in a population with a high risk of PCa, the mpMRI can even reach a NPV of $88 \%$. The patient's baseline risk must be valuated and there are calculators used to determine the patient's risk (by example, Prostate Cancer Risk Calculators) (16).

Long-term NPV has been addressed in a recent study: $84 \%$ of patients with negative mpMR were free of cancer even after 4 years of follow-up, whereas 16\% developed PCa although only $4 \%$ developed a csPCa (17).

\section{Characterization of the familiar history and genetic}

There are men with low PSA level, negative mpMRI and unpalpable disease with PCa (18). These men have usually familiar history of PCa and undergo premature serial PSA exams, clinic visits, and rectal examination.

The genetic tests are suggested in men with one brother or father or two or more male relatives with one of the following: diagnosed with $\mathrm{PCa}$ at age less than 60 years (recommended), any of whom died of PCa (recommended), any of whom had metastatic PCa (recommend) (19). Genetic testing should be considered in men with family history of 2 or more cancers in hereditary breast and ovarian cancer, or Lynch syndrome in any relatives on the same of the family (especially if diagnosed at age $<50$ years) (20). The BRCA2 mutation leads to a three times greater risk of death (21).

There are many other important aspects of genetics that have been identified over the years by numerous studies. First, carriers of BRCA1 and mostly BRCA2 mutations are at high risk of developing PCa. Germline mutations in the BRCA genes, mainly in BRCA2, also have implications in the prognosis and management of the disease, with medi- 
an cause-specific survival (CSS) for carriers of 2.1 years compared with 12.4 years for non-carriers (22).

Secondly, Lynch syndrome is a type of inherited cancer syndrome associated with germline mutations mainly in the MSH2 and MSH6 genes; affected individuals are at an increased risk for colorectal cancer but also for other associated tumors including utero and ovary, stomach and small bowel, pancreas, ureter or renal pelvis, biliary tract, brain and prostate. The risk of developing PCa in this patients is twofold higher than general population (23). Criteria of hereditary PCa from John Hopkins University (JHU) included at least 3 first-line relatives with PCa or cases of PCa in three generations, as well as 2 relatives with $\mathrm{PCa}<56$ years of age (24). Approximately $8 \%$ of young PCa patients have hereditary PCa. The recommendation of the National Comprehensive Cancer Network (NCCN) is to use Next Generation Sequencing to sequence the multigene panel, which includes at least BRCA1, BRCA2, ATM, BRIP1, CHEK2, NBN, HOXB13, MLH1, MSH2, MSH6 and PMS2 (25).

Men with age between $45-75$ years with PSA $<1 \mathrm{ng} / \mathrm{mL}$, DRE normal or PSA $1-3 \mathrm{ng} / \mathrm{ml}$ and DRE normal are often underestimated, this led to correctly frame the patient who needs annual checks for PCa early detection. However, a personalised risk-adapter strategy for early detection PCa could be associated with an important risk reduction of over-diagnosis and over-biopsy $(26,27)$.

\section{Pathological point of view: Definition of csPCa}

Although Epstein criteria have a suboptimal accuracy for predicting significant $\mathrm{PCa}$, the pathological concept of significant prostate cancer is a lesion with volume of $>$ $0.2 \mathrm{ml}$, a Gleason Grade $\geq 7$ or extraprostatic extension (28), The index lesion is defined as the larger and/or containing high grade tumor (pattern 4 or 5) (29).

These values were defined assessing 185 patients who underwent radical prostatectomy with a median of five years follow-up in which Epstein et al. demonstrated that none of the patients with palpable tumors clinical T2 with a volume $<0.2 \mathrm{cc}$ had extra-prostatic extension (EPE) or biochemical recurrence (BCR) (30). However, there was a high incidence of multifocal PCa and consequently summing the volumes of many small prostate tumors would result in a total volume higher than $0.2 \mathrm{ml}$, although actually there is no evidence that these smaller multifocal tumors impact on the prognosis of the index tumor (31). In the era of cognitive guided fusion biopsies, in the last years several studies showed that imaging driven biopsy help to target the cancer properly increasing the grading assessment through biopsy $(32,33)$. PCa is considered a paradigm of morphologic heterogeneity and the role of the pathologist and of biopsy technique assumes great importance for the choice of treatment and appropriate follow-up (26). Grade Group 1 (Gleason score 3+3=6), pathologically composed of individual discrete wellformed separated glands, is very homogeneous with an excellent prognosis $(34,35)$.

In a population of 20845 men with clinically localized $\mathrm{PCa}$ treated by radical prostatectomy in four different American Hospitals, Epstein et al. did not observe distant metastasis or cancer-specific mortality in a subgroup of more than 6000 men with organ-confined and negative margins with pure Gleason score 6 disease; the risk of progression in this group is approaching 0\% (36). Despite these results, we must consider that not all tumors that "look good" from a clinical point of view $(\mathrm{PSA}<3 \mathrm{ng} / \mathrm{ml}$, negative DRE, negative transrectal ultrasound, PI-RADS 3 at mpMR) are also good from the histological and molecular point of view. Kamoun et al. subdivided prostate tumors in three different genetical signatures profiled on DNA methylation, SNP arrays and mRNA arrays. Molecular subtype "S2" tumors, which mainly included ISUP group 1 and 2, present with TMPRSS2-ERG fusions with constancy in other molecular subgroups and were also characterized by an almost total absence of mutations in PTEN, in the phosphoinositol kinase pathway and in p53. However a little percentage of ISUP 3 and 4 tumors was represented also in this class (37).

\section{Cribriform histology and intraductal carcinoma}

While pure Gleason score 6 prostate cancers have an excellent outcome in terms of progression, absence of distant metastasis and disease-free survival, areas of cribriform pattern 4 histology, intraductal carcinoma and PCa with reactive stroma are characterized by aggressive genetic alterations and worse oncological outcomes.

Presence of pattern 4 with cribriform histology must be reported because its presence and its quantitative representation (in terms of percentage) aggravate prognosis (38). Cribriform can be confused by an inexperienced pathologist with other histotypes like glomeruloid glands, fused glands and poorly formed glands. Hollemans et al. distinguished cribriform type into two groups: small cribriform (< 12 lumina) and large cribriform ( $>12$ lumina): patients with large invasive cribriform growth belong to a more aggressive subgroup with an increased risk for biochemical recurrence and metastasis (39). Intraductal carcinoma (IDC-P) is pathologically recognized by its cribriform or solid growth pattern distending preexistent acini and prostatic ducts with preservation of basal cells; in most cases IDC-P is closed associated to presence of high grade PCa and high volume cancers (40). Percentage of grade 4 , grade 4 cribriform pattern and the presence of intraductal carcinoma at biopsy have an important impact on the prognosis. The presence of intraductal growth or invasive cribriform cancer at biopsy outperforms percentage Grade 4 in predicting outcome of Gleason score $3+4=$ 7 PCa: in 370 men with GS $3+4=7$ prostatic cancer was demonstrated that invasive cribriform and/or intraductal carcinoma are independent parameters for BCR after radical prostatectomy, while percentage of Gleason group 4 is not. The majority of IDC-P derived from adjacent highgrade invasive carcinoma via retrograde spreading of cancer cells along benign ducts and acini; a small group of IDC-P may represent the transformation of precancerous intraductal proliferation induced by various oncogenic alterations (41).

The prevalence of IDC-P increased significantly from $2.1 \%$ in low-risk patient up to $56.0 \%$ in metastatic or recurrent $\mathrm{PCa}$ patients (42). Interestingly, in patients treated with androgen deprivation therapy (ADT) or chemotherapy, IDC-P was reported in $60 \%$ of PCa indicating that IDC-P may correlate with the development of castration-resistant prostate cancer. 


\section{PCa with reactive stroma}

Another important overview is focused on PCa with reactive stroma (also called stromogenic PCa), that is, by definition, composed by at least $50 \%$ reactive stroma (stroma/epithelium ratio $\geq 1$ ). The prototypic "stromogenic" carcinoma is of the classic acinar subtype, with well-formed glands surrounded by an evident amount of stroma. This pathological feature is correlated with a worse prognosis; in samples with high reactive stroma, metabolites and genes linked to immune functions and extracellular matrix remodeling are significantly upregulated (43).

\section{PSA - Negative prostatic tumors}

PSA has been used as serum marker for PCa screening in the male population, but its specificity is low due to its production by normal epithelial tissue. PSA antibody is currently used in immunohistochemistry to determine whether tumor masses of unknown origin can be assigned to a prostate cancer. However, some prostatic tumors do not express PSA in immunohistochemistry and do not determine increases in PSA levels in the blood (34). In poorly differentiated tumors or metastatic foci, PSA expression might be decreased or even lost. PCa with neuroendocrine differentiation often lost PSA expression (44). More specific markers such as PSMA and NKX3.1 are now available to overcome the limited sensitivity of PSA (Figure 1) (45). Moreover, other tumor types, although rare, such as prostatic stromal and smooth muscle tumors, both benign and malignant, solitary fibrous, neural, germ cell, hematopoietic and melanocytic tumors, can form a palpable mass in the prostate, without giving an increase of the serum PSA (46). PSA screening alone is not sufficient to exclude a prostatic tumor; a physical inspection with digital rectal examination is highly recommended and should not be replaced by a PSA screening alone.

\section{Figure 1.}

Hematoxylin \& Eosin-stained section of a prostate cancer bone metastasis (A) with a focal expression of PSA (B) and a diffuse and strong expression of PSMA (C) and NKX 3.1 (D).

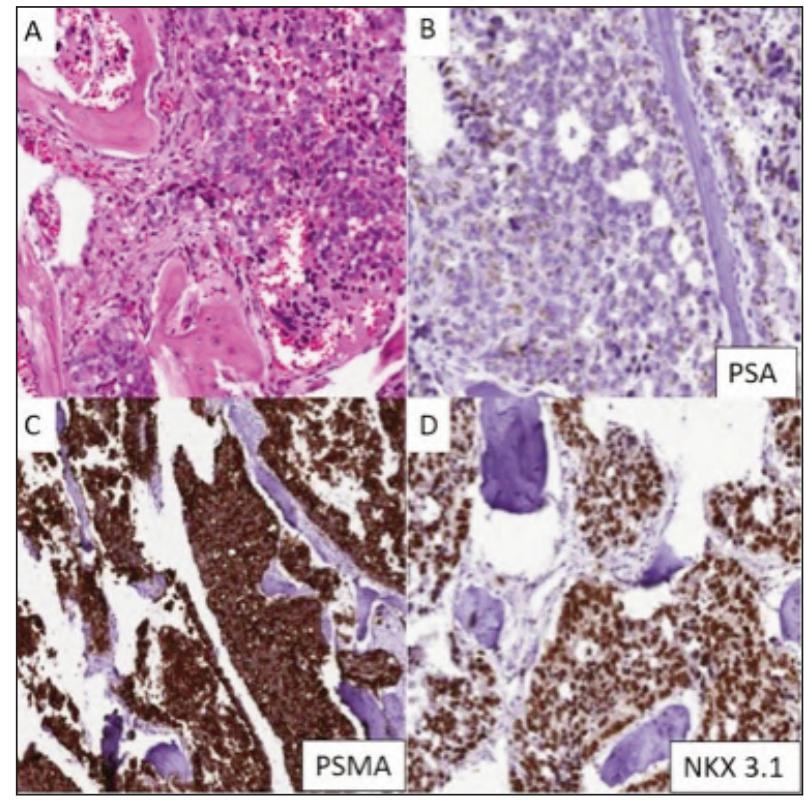

\section{The role of $\mathrm{mpMR}$}

According to the most recent update of the European Urology Association Guidelines, the introduction of mpMR in the diagnostic pathway in all patients with PSA elevation leads to significant improvement of clinically significant PCa detection (2). The lesion detected by imaging is then targeted by biopsies under the guide of MRI/ultrasound fusion technology. This increases the detection of csPCa, lowering the detection of insignificant disease. Also "biopsy naïve" patients have a benefit with a MR evaluation. However, it is of outmost importance that a negative MR does not exclude the possibility of significant PCa. Moreover, deciding whether or not is safe to avoid a biopsy in a naive patient with negative imaging should also rely on PSAD, digital rectal evaluations, nomograms, new biomarkers and family history (10).

The PROMIS study showed that the use of mpMRI reduces of a $5 \%$ the detection rate of indolent cancers lowering the need of biopsy in 27\% of patients (47). Moreover, the application of mpMR/TRUS fusion biopsy technology increased of a $18 \%$ the rate of diagnosis of csPCa compared to the traditional systematic biopsy. The need of systematic biopsy is underlined by several studies that reported a significant percentage (5\% up to 16\%) of csPCa missed by MR-guided biopsy.

Results found by Rouvière et al. highlighted the role of systematic biopsy to detect csPCa in men with abnormal PSA and negative imaging/DRE (48). They stated that the mpMR in biopsy naive patients can improve csPCa detection rate using target biopsy plus concurrent systematic biopsy. Any differences in detection rate of high grade cancers (ISUP grade $\geq 2$ ) was observed between targeted and systematic biopsies, but they found a significant improvement (plus 5\% up to 7\%) using the combination of both techniques (48).

Are we ready to avoid biopsy in negative $m p M R$ ?

Yes, since the benefit is reduced in both detection of indolent disease and number of men undergoing biopsy. However, from $5 \%$ to $20 \%$ of csPCa have negative mpMR imaging (49).

This rate of undetected csPCa should guide the clinician to the best tailored strategy for the single patient balancing monitoring versus early biopsy approach in terms of morbidity of biopsy and delayed cancer diagnosis.

In patients with PSA below the limit and normal DRE, the mpMR is not indicated and not supported by Guidelines (2). Therefore, based on available knowledge, the use of $\mathrm{mpMR}$ as a screening tool in patients with normal PSA and negative DRE must be discouraged. The negative predictive value (NPV) of mpMR changes significatively in relation to the risk of $\mathrm{PCa}$ in a population, where the incidence of cancer is low (normal PSA) the diagnostic performance dropped significatively to $50 \%$, while in high risk is up $88 \%$. To improve the NPV of mpMR in men with normal PSA, other factors such as genetic, PSAD or PSAV should be evaluated to select those men with higher risk to have cancer (50).

A recent and very interesting study estimated the risk to detect cancer over the time after a negative mpMR at baseline (17). After 4 years of follow-up, they found 84\% of patients remained free of cancer and overall 16\% 
developed PCa but only 4\% csPCa. Although of its several limitations (e.g. control group, highly selected cases in referral centre), this study shows that a negative mpMR predict a lower risk over the time to develop csPCa: 4\% (1 man every 20) compared to $8 \%$ of calculated risk for a man to be diagnosed with clinically significant PCa throughout his lifetime.

\section{Limits of $m p M R$}

Despite the wide acknowledged improvements cited in the previous paragraph and being advocate as the reference standard for prostate radiological imaging, $\mathrm{mpMR}$ is not exempt from drawbacks. According to Quon et al., mpMR missed up to $20 \%$ of csPCa lesions or underestimated its size (51). After a second-look of MR imaging, $58 \%$ of the missed lesions were not confirmed as benign findings. In fact, this technique needs further implementations to reduce inter-observer variability and to reduce false negative rates.

The radiologist's experience is shown to be a key factor in imaging interpreting and $\mathrm{PCa}$ recognition. A recent study analysed the different reports of 9 radiologists on the same mpMRI by comparing their experience (52). Among PI-RADS 2 results, the possibility of finding clinically significant $\mathrm{PCa}$ in the biopsy ranged from 15 to $35 \%$. The detection of any type of PCa in PI-RADS 2 ranged from 15 to $75 \%$. In 2018 Johnson et al. analysed the mpMRI performed on 588 patients before radical prostatectomy, using a 3 Tesla MRI in a single institution with expert radiologists (48). Overall, the mpMRI detected 541 out of 1213 pathologic lesions, which represent a sensitivity of $45 \%$, a positive predictive value of $81 \%$ and a false positive rate of $19 \%$. Therefore larger (>0.5 $\mathrm{ml}$ ), high grade and solitary tumors were more likely to be detected. Comparing mpMR and surgical specimens, $90 \%$ of the non-csPCa were missed, but also the $40 \%$ of Gleason Score 3+4, the 26\% of Gleason Score 4+3 and the $22 \%$ of Gleason Score $\geq 8$.

In a recently published review, Chatterjee et al. found that the inter-observer variability and lack of standardisation in reporting radiological findings are the most important interpretative drawbacks (53).

They described a variety of interpretative and technical pitfalls that influenced negatively mpMR performances: 1) different anatomic features can simulate PCa such as the anterior fibromuscular stroma, the periprostatic venous plexus and the pseudocapsule of the Transitional Zone (TZ); 2) others histologic benign conditions such as inflammation and prostatitis, could mimic PCa; 3) postbiopsy haemorrhage, prostatic calcifications associated with benign prostatic hyperplasia nodules in the transitional zone; 4) technical artifacts or image distortion due to endorectal coil placements and motion artefacts.

The low-intensity T2 signal and the heterogeneity of enhancement patterns in the peripheral zone are reported to reduce the diagnostic yield of mpMR (53).

Improvements of MR imaging: PI-RADS 2.1

The first version of the Prostate Imaging Reporting and Data System (PI-RADS v1) released in 2012 by the European Society of Urogenital Radiology and then updated to Version 2.0 in 2015 has been considered and adopted as the reference standard tool to report and early detect PCa. The most reported limit of this scoring system is the inter-reader concordance ratio (moderate), which limits the diagnostic performance of this system. Moreover, despite technological improvements, the study of the transition zone remains challenging if compared to the peripheral zone due to the presence of benign hyperplasia as a mimic of PCa (2).

In 2019 the PI-RAD system was updated to version 2.1 with leading changes regarding the transition zone mpMRI conundrum (54). The most important ones are: 1) the definition of atypical nodules in the TZ and the downgrading to category score 1 of the completely encapsulated nodules (encapsulated or homogeneous circumscribed defined as PI-RADS 2 in the previous category system); 2) the characterisation of these nodules trough diffusion weighted imaging (DWI) features to improve detection and localization of PI-RADS 3 areas.

Focus on PI-RADS 3 lesions in the TZ vs PZ

MRI lesions PI-RADS 3 are termed as "equivocal" for the presence of clinically csPCa. PI-RADS 3 represents a "grey zone" that needs to be further investigated to solve the issue of biopsy or not biopsy. They could be malignant lesions but mostly they are benign lesions or nonsignificative cancer (55).

The PI-RADS version 2 uses a 5-point scale based on the likelihood (probability) that a combination of mpMR findings on T2-weighted imaging (T2W), diffusion-weighted imaging (DWI), and dynamic contrast enhancement (DCE) correlates with the presence of a clinically significant cancer depending on cancer volume $(>0.5 \mathrm{ml})$, location and background tissue within the prostate gland.

The Peripheral Zone (PZ) of the prostate has a high risk of develop prostate cancer compared to the Transizion Zone (TZ). Therefore, doubtful MR imaging (defined as PIRADS 3) has different diagnostic value taken into account prostatic anatomical zones. This is supported by Yang et al. who analyzed cancer detection rate in 683 patients with PI-RADS 3 lesions of the PZ and TZ (56). They reported $37 \%$ cancer detection and $18.7 \%$ of csPCa in the PZ, while in the TZ the overall cancer detection was $16.4 \%$ and the rate of csPCa was $6.0 \%$.

Furthermore, using a PSAD greater than 0.15 and age greater than 68 years, they calculated that $24 \%$ of biopsy could be omitted by losing only one csPCa, with a sensitivity of $80 \%$ and a negative predictive value of $92.3 \%$. They conclude that significant cancer is uncommon in TZ and the active surveillance is the optimal choice, especially among patients without risk factors such as those with low PSAD.

Many studies report that mpMR is very reliable as a negative predictive value, but it depends on prevalence of disease in the population studied and the a priori risk of developing disease. Since, PI-RADS 3 does not identify the same risk of cancer in different prostate zones, the predictive value of PI-RADS 3 should be considered as low in the $\mathrm{TZ}$ respect to $\mathrm{PZ}$.

However, the estimating of the predictive value of PIRADS 3 lesions has methodological bias because it do not represent the primary endpoint of published studies, 
as Maggi et al. very recently underlined after an extensive review (57). In 28 studies with 10.176 patients $(56.5 \%$ with $\mathrm{PCa}$ and $40.0 \%$ with csPCa), 1,759 men with targeted biopsies on PI-RADS 3 lesions were retrieved: the overall rate of $\mathrm{csPCa}$ detection was $36 \%$ and $18.5 \%$.

The combination technique (targeted and systematic biopsy) has the better detection rate of $37 \%$ versus the $24 \%$ of exclusive targeted biopsy. There is no significantly relevant difference between version 1 and version 2 of PI-RADS in the detection of prostate cancer (57). However, they did not differentiate the cancer detection comparing prostatic zones.

Byun et al. evaluated the PI-RADS v2.1 diagnostic performances and inter-reader agreement on the PCa detection in the $\mathrm{TZ}$ comparing results with the previous version (58). Their results demonstrated that the last version has both higher sensitivity and specificity for the overall detection of PCa for category higher than 3 lesions, independently of the prostatic zone. Furthermore, they reported a reduction of proportion of category 3 lesions, while the detection rates of $\mathrm{csPCa}$ at this cut-off value significantly increased accordingly with the inter-reader agreement. Same findings were confirmed by Wang et al. that also suggested that PI-RADS version 2.1 improves the overall detection rates of $\mathrm{PCa}$, specifically in the $\mathrm{TZ}$ zone, compared to v2.0 (54).

Recently, several studies evaluated the role of PSAD as predictive variable to predict csPCa in PI-RADS 3 lesions: a cut-off $0.15 \mathrm{ng} / \mathrm{ml} / \mathrm{cc}$ was the most significant positive predictive value for detect csPCa. Hansen and Ullrich et al. confirm that the choice to execute biopsies in the group of PI-RADS 3 with PSAD > $0.15 \mathrm{ng} / \mathrm{ml} / \mathrm{cc}$ permit to avoid $53.4 \%$ of biopsies in this population of patients $(59,60)$. Also Venderink et al. calculated that $42 \%$ patients with PI-RADS 3 lesions and PSAD less than 0.15 could avoid biopsy with the loss of only $6 \%$ of cs PCa $(95 \%$ C.I. 2-15) (61). Lowering the cut-off to 0.12 they spared $26 \%$ of avoidable biopsies without losing any csPCa (61).

Schoots et al. reviewed the probability of csPCa in PI-RADS 3 lesions observed in biopsy naive, previous negative biopsy, and active surveillance patients: they retrieved $21 \%, 16 \%$ and $17 \%$ of csPCa, respectively (62). They focused on size of the lesion: PI-RADS 3 lesions in both peripheral zone (PZ) and transition zone (TZ) greater than $10 \mathrm{~mm}$ should be re-classified as PI-RADS 4 according to their results. Other than diameter, the decision to proceed with the biopsy must include clinical elements such as PSAD, PSAV and previous biopsies.

The risk of missing csPCa must be discussed with the patients and balanced

\section{Figure 3.}

Clinical pathway in biopsy negative patient with PI-RADS.

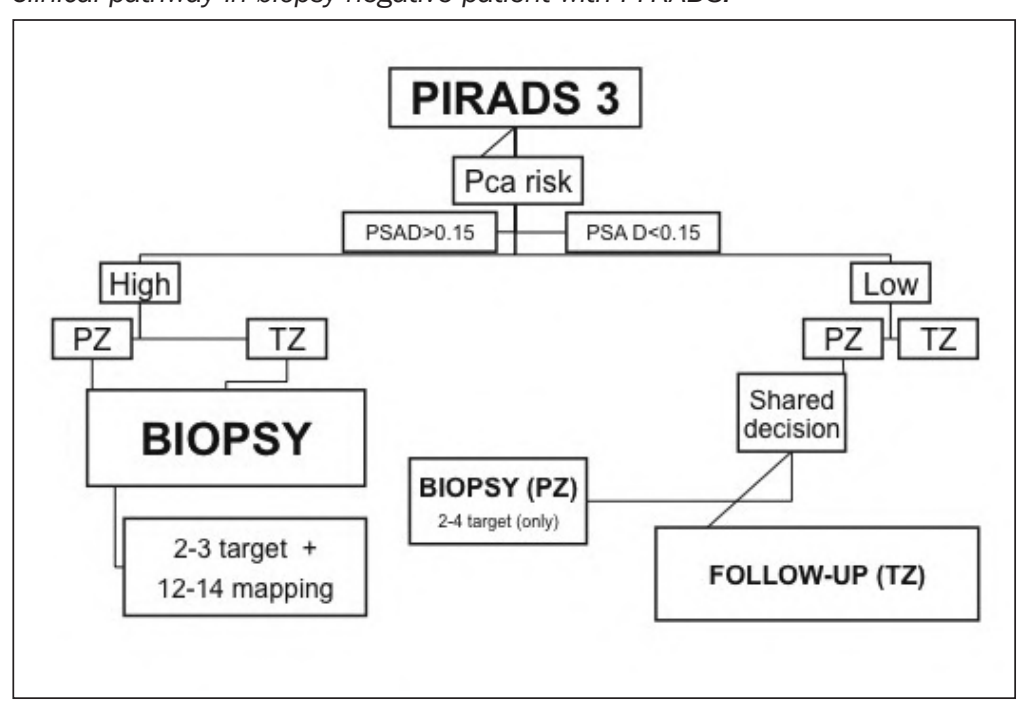

against saving biopsies (side effects) and overdiagnosing of insignificant PCa on an individual basis.

Van Der Sar et al. investigated retrospectively outcomes such as window of curability and complication and costs of clinical surveillance compared to immediate biopsy (63). All outcomes were not influenced by both strategies as well as the risk profile of the cancers appeared similar.

The large part of patients preferred clinical surveillance (57\%) compared to immediate biopsy (43\%). Frye et al. monitoring PI-RADS 3 lesions in active surveillance reported a rate of progression of $20 \%$ precisely on the area of the target lesion PI-RADS 3 compared to $29 \%$ of overall progression (64)

Therefore, we can assume that defer the biopsy in PIRADS 3 lesion in the TZ can be a safe strategy, as we suggested in a flow-chart summarized in the Figures 2, 3.
Figure 2.

Clinical pathway in biopsy naive patient with PI-RADS 3 lesions.

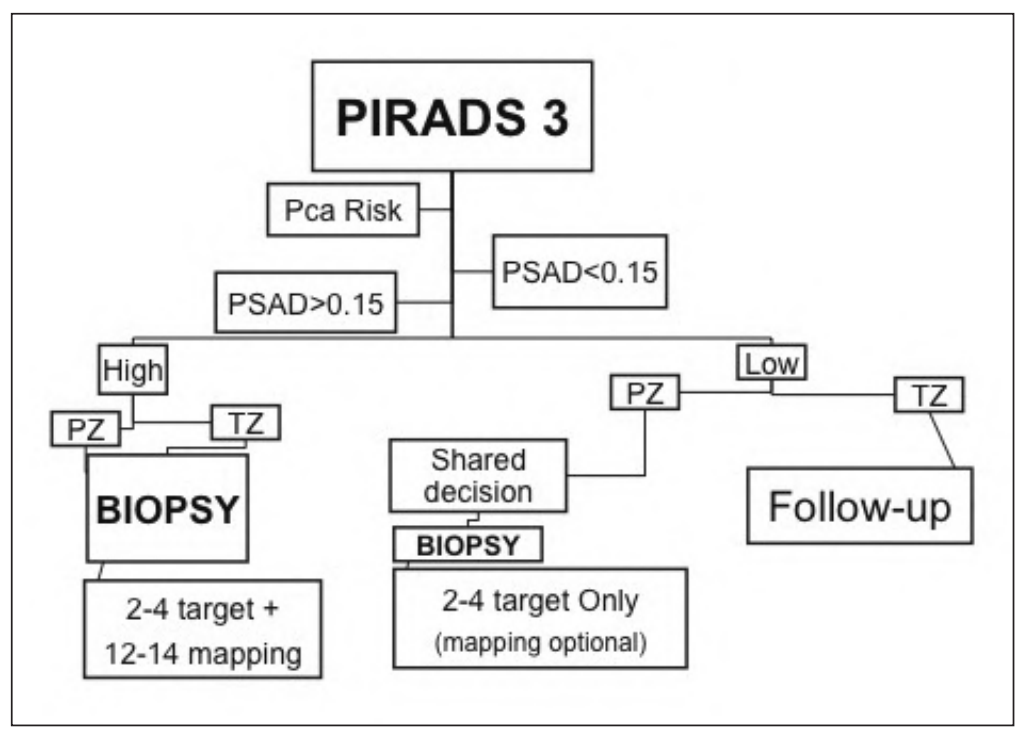


In conclusion the management PI-RADS 3 lesion should be based on shared decision to immediate biopsy or follow-up considering the following points: 1) estimation of prostate cancer risk based on nomograms and risk calculators, 2) use carefully mpMR in patients with low risk of csPCa and normal PSA, 3) MR should be done in quality checked centers (controlled incidence of PI-RADS 3 lesions and correlation between radiological and pathological finding), 3) lower urinary tract symptoms 4) use of PSAD, anatomical zone (TZ vs $\mathrm{PZ}$ ), and family history.

All those factors should be taken into account and patient should be involved in the final decision to perform a fusion prostate biopsy.

\section{ReFERENCES}

1. Cimadamore A, Cheng M, Santoni M, et al. New prostate cancer targets for diagnosis, imaging, and therapy: focus on prostate-specific membrane antigen. Front Oncol. 2018; 8:653.

2. Mottet N, Bastian P, Bellmunt J, et al. EAU - EANM - ESTRO ESUR - SIOG: Guidelines on Prostate Cancer. Eur Assoc Urol. 2020; 1-182.

3. Lambert E, Goossens M, Palagonia E, et al. Changes in serum PSA after endoscopic enucleation of the prostate are predictive for the future diagnosis of prostate cancer. World J Urol. 2020 Sep 30. doi: 10.1007/s00345-020-03444-0. Epub ahead of print.

4. Thompson IM, Pauler DK, Goodman PJ, et al. Prevalence of prostate cancer among men with a prostate-specific antigen level < or $=4.0 \mathrm{ng}$ per milliliter. N Engl J Med. 2004; 350:2239-46.

5. Maselli G, Tucci G, Mazzaferro D, et al. Prolonged antibiotic therapy increases risk of infection after transrectal prostate biopsy: a case report after pancreasectomy and review of the literature. Arch Ital Urol Androl. 2014; 86:387-8.

6. Halpern JA, Oromendia C, Shoag JE, et al. Use of digital rectal examination as an adjunct to prostate specific antigen in the detection of clinically significant prostate cancer. J Urol. 2018; 199:947-53.

7. Vickers AJ, Ulmert D, Sjoberg DD, et al. Strategy for detection of prostate cancer based on relation between prostate specific antigen at age 40-55 and long term risk of metastasis: Case-control study. BMJ. 2013; 346:f2023.

8. Carter HB, Ferrucci L, Kettermann A, et al. Detection of lifethreatening prostate cancer with prostate-specific antigen velocity during a window of curability. J Natl Cancer Inst. 2006; 98:15217.

9. Vickers AJ, Till C, Tangen CM, et al. An empirical evaluation of guidelines on prostate-specific antigen velocity in prostate cancer detection. 2011; 103:0-7.

10. Fandella A, Scattoni V, Galosi A, et al. Italian Prostate Biopsies Group: 2016 Updated Guidelines Insights. Anticancer Res. 2017; 37:413-24.

11. Martino P, Galosi AB, Bitelli $M$, et al. Practical recommendations for performing ultrasound scanning in the urological and andrological fields. Arch Ital Urol Androl. 2014; 86:56-78.

12. Pagniez MA, Kasivisvanathan V, Puech P, et al. Predictive factors of missed clinically significant prostate cancers in men with negative magnetic resonance imaging: a systematic review and metaanalysis. J Urol. 2020; 204:24-32.
13. Roscigno M, Stabile A, Lughezzani G, et al. The use of multiparametric magnetic resonance imaging for follow-up of patients included in active surveillance protocol. Can PSA density discriminate patients at different risk of reclassification? Clin Genitourin Cancer. 2020; 18:e698-704.

14. Bhat NR, Vetter JM, Andriole GL, et al. Magnetic resonance imaging-defined prostate-specific antigen density significantly improves the risk prediction for clinically significant prostate cancer on biopsy. Urology. 2019; 126:152-7.

15. Moldovan PC, Van den Broeck T, Sylvester R, et al. What is the negative predictive value of multiparametric magnetic resonance imaging in excluding prostate cancer at biopsy? A systematic review and meta-analysis from the European Association of Urology Prostate Cancer Guidelines Panel. Eur Urol. 2017; 72:250-66.

16. Poyet C, Nieboer D, Bhindi B, et al. Prostate cancer risk prediction using the novel versions of the European Randomised Study for Screening of Prostate Cancer (ERSPC) and Prostate Cancer Prevention Trial (PCPT) risk calculators: independent validation and comparison in a contemporary European cohort. BJU Int. 2016; 117:401-8

17. Panebianco V, Barchetti G, Simone G, et al. Negative multiparametric magnetic resonance imaging for prostate cancer: what's next? Eur Urol 2018; 74:48-54.

18. Andreoiu M, Cheng L. Multifocal prostate cancer: biologic, prognostic, and therapeutic implications. Hum Pathol 2010; 41:781-93

19. Giri VN, Knudsen KE, Kelly WK, et al. Implementation of germline testing for prostate cancer: Philadelphia Prostate Cancer Consensus Conference 2019. J Clin Oncol. 2020; 38:2798-811.

20. Zhen JT, Syed J, Nguyen KA, et al. Genetic testing for hereditary prostate cancer: current status and limitations. Cancer. 2018; 124:3105-17.

21. Oh M, Alkhushaym N, Fallatah S, et al. The association of BRCA1 and BRCA2 mutations with prostate cancer risk, frequency, and mortality: A meta-analysis. Prostate. 2019; 79:880-95.

22. Castro E, Eeles R. The role of BRCA1 and BRCA2 in prostate cancer. Asian J Androl. 2012; 14:409-14.

23. Raymond VM, Mukherjee B, Wang F, et al. Elevated risk of prostate cancer among men with lynch syndrome. J Clin Oncol. 2013; 31:1713-8.

24. Ostrander EA, Stanford JL. Genetics of prostate cancer: too many loci, too few genes. Am J Hum Genet. 2000; 67:1367-75.

25. Carroll PR, Parsons JK, Andriole G, et al. Prostate cancer early detection, version 2.2015: clinical practice guidelines in oncology. J Natl Compr Cancer Netw. 2015; 13:1534-61.

26. Gasparrini S, Cimadamore A, Mazzucchelli R, et al. Pathology and molecular updates in tumors of the prostate: towards a personalized approach. Expert Rev Mol Diagn. 2017; 17:781-9.

27. Dell'Atti L, Galosi AB. Safety of transrectal ultrasound-guided prostate biopsy in patients affected by Crohn's disease. Arch Ital Urol Androl. 2017; 89:106-9.

28. Oon SF, Watson RW, O'Leary JJ, Fitzpatrick JM. Epstein criteria for insignificant prostate cancer. BJU Int. 2011; 108:518-25.

29. Mazzucchelli R, Galosi AB, Lopez-Beltran A, et al. Pathological issues in biopsy specimens of men with prostate cancer eligible for active surveillance. Arch Ital di Urol Androl. 2014; 86:314-8. 
30. Epstein JI, Egevad L, Amin MB, et al. The 2014 International Society of Urological Pathology (ISUP) Consensus Conference on Gleason grading of prostatic carcinoma: definition of grading patterns and proposal for a new grading system. Am J Surg Pathol. 2016; 40:244-52.

31. Matoso A, Epstein JI. Defining clinically significant prostate cancer on the basis of pathological findings. Histopathology. 2019; 74:135-45.

32. Lacetera V, Cervelli B, Cicetti A, et al. MRI/US fusion prostate biopsy: Our initial experience. Arch Ital di Urol Androl. 2016; 88:296-9.

33. Galosi AB, Maselli $G$, Sbrollini $G$, et al. Cognitive zonal fusion biopsy of the prostate: Original technique between target and saturation. Arch Ital Urol Androl. 2016; 88:292-5.

34. Cimadamore A, Scarpelli M, Raspollini MR, et al. Prostate cancer pathology: What has changed in the last 5 years. Urologia. 2020; 87:3-10.

35. Montironi R, Santoni M, Mazzucchelli R, et al. Prostate cancer: from Gleason scoring to prognostic grade grouping. Expert Rev Anticancer Ther. 2016; 16:433-40.

36. Magi-Galluzzi C, Montironi R, Epstein JI. Contemporary Gleason grading and novel Grade Groups in clinical practice. Curr Opin Urol. 2016; 26:488-92.

37. Kamoun A, Cancel-Tassin G, Fromont G, et al. Comprehensive molecular classification of localized prostate adenocarcinoma reveals a tumour subtype predictive of non-aggressive disease. Ann Oncol. 2018; 29:1814-21.

38. Sauter G, Steurer S, Clauditz TS, et al. Clinical utility of quantitative gleason grading in prostate biopsies and prostatectomy specimens. Eur Urol. 2016; 69:592-8.

39. Hollemans E, Verhoef EI, Bangma CH, et al. Large cribriform growth pattern identifies ISUP grade 2 prostate cancer at high risk for recurrence and metastasis. Mod Pathol. 2019; 32:139-46.

40. Roberts JA, Zhou M, Park YW, Ro JY. Intraductal carcinoma of prostate: A comprehensive and concise review. Korean J Pathol. 2013; 47:307-15.

41. Zong Y, Montironi R, Massari F, et al. Intraductal carcinoma of the prostate: pathogenesis and molecular perspectives. Eur Urol Focus 2020:S2405-4569(20)30291-1

42. Porter LH, Lawrence MG, Ilic D, et al. Systematic review links the prevalence of intraductal carcinoma of the prostate to prostate cancer risk categories. Eur Urol. 2017; 72:492-5.

43. Silva MM da J, Matheus WE, Garcia PV, et al. Characterization of reactive stroma in prostate cancer: involvement of growth factors, metalloproteinase matrix, sexual hormones receptors and prostatic stem cells. Int Braz J Urol. 2015; 41:849-58.

44. Wang W, Epstein JI. Small cell carcinoma of the prostate. A morphologic and immunohistochemical study of 95 cases. Am J Surg Pathol. 2008; 32:65-71.

45. Abouhashem NS, Salah S. Differential expression of NKX 3.1 and HOXB 13 in bone metastases originating from prostatic carcinoma among the Egyptian males. Pathol Res Pract. 2020; 216:153221.

46. Paner GP, Aron M, Hansel DE, Amin MB. Non-epithelial neoplasms of the prostate. Histopathology. 2012; 60:166-86.
47. Ahmed HU, El-Shater Bosaily A, et al. Diagnostic accuracy of multi-parametric MRI and TRUS biopsy in prostate cancer (PROMIS): a paired validating confirmatory study. Lancet. 2017; 389:815-22.

48. Rouvière O, Puech P, Renard-Penna R, Claudon M, Roy C, Mège-Lechevallier $F$, et al. Use of prostate systematic and targeted biopsy on the basis of multiparametric MRI in biopsy-naive patients (MRI-FIRST): a prospective, multicentre, paired diagnostic study. Lancet Oncol. 2019; 20:100-9.

49. Johnson DC, Raman SS, Mirak SA, Kwan L, Bajgiran AM, Hsu W, et al. Detection of individual prostate cancer foci via multiparametric magnetic resonance imaging. Eur Urol. 2019; 75:712-20

50. Galosi AB, Dell'Atti L, Bertaccini A, Gion M, Francavilla S, Ferretti $S$, et al. Clinical evaluation of the iXip index to reduce prostate re-biopsies. Cancer Treat Res Commun. 2018; 16:59-63.

51. Quon JS, Moosavi B, Khanna M, Flood TA, Lim CS, Schieda N. False positive and false negative diagnoses of prostate cancer at multi-parametric prostate MRI in active surveillance. Insights Imaging. 2015; 6:449-63.

52. Sonn GA, Fan RE, Ghanouni P, Wang NN, Brooks JD, Loening $A M$, et al. Prostate magnetic resonance imaging interpretation varies substantially across radiologists. Eur Urol Focus. 2019; 5:592-9.

53. Chatterjee A, Thomas S, Oto A. Prostate MR: pitfalls and benign lesions. Abdom Radiol 2020; 45:2154-64.

54. Wang Z, Zhao W, Shen J, Jiang Z, Yang S, Tan S, et al. PI-RADS version 2.1 scoring system is superior in detecting transition zone prostate cancer: a diagnostic study. Abdom Radiol. 2020; 45:41424149.

55. Weinreb JC, Barentsz JO, Choyke PL, Cornud F, Haider MA, Macura KJ, et al. PI-RADS Prostate Imaging - Reporting and Data System: 2015, Version 2. Eur Urol. 2016; 69:16-40.

56. Yang S, Zhao W, Tan S, Zhang Y, Wei C, Chen T, et al. Combining clinical and MRI data to manage PI-RADS 3 lesions and reduce excessive biopsy. Transl Androl Urol. 2020; 9:1252-61.

57. Maggi M, Panebianco V, Mosca A, Salciccia S, Gentilucci A, Di Pierro G, et al. Prostate imaging reporting and data system 3 category cases at multiparametric magnetic resonance for prostate cancer: a systematic review and meta-analysis. Eur Urol Focus. 2020; 6:463-78.

58. Byun J, Park KJ, Kim M hyun, Kim JK. Direct comparison of PIRADS version 2 and 2.1 in transition zone lesions for detection of prostate cancer: preliminary experience. J Magn Reson Imaging. 2020; 52:577-86.

59. Hansen NL, Kesch C, Barrett T, Koo B, Radtke JP, Bonekamp $D$, et al. Multicentre evaluation of targeted and systematic biopsies using magnetic resonance and ultrasound image-fusion guided transperineal prostate biopsy in patients with a previous negative biopsy. BJU Int. 2017; 120:631-8.

60. Ullrich T, Quentin M, Arsov C, Schmaltz AK, Tschischka A, Laqua N, et al. Risk stratification of equivocal lesions on multiparametric magnetic resonance imaging of the prostate. J Urol. 2018; 199:691-8.

61. Venderink W, van Luijtelaar A, Bomers JGR, van der Leest $M$, Hulsbergen-van de Kaa C, Barentsz JO, et al. Results of targeted biopsy in men with magnetic resonance imaging lesions classified 
equivocal, likely or highly likely to be clinically significant prostate cancer. Eur Urol. 2018; 73:353-60.

62. Schoots IG. MRI in early prostate cancer detection: how to manage indeterminate or equivocal PI-RADS 3 lesions? Transl Androl Urol. 2018; 7:70-82.

63. van der Sar ECA, Kasivisvanathan V, Brizmohun M, Freeman $A$, Punwani S, Hamoudi $R$, et al. Management of radiologically indeterminate magnetic resonance imaging signals in men at risk of prostate cancer. Eur Urol Focus. 2019; 5:62-8.

64. Frye TP, George AK, Kilchevsky A, Maruf M, Siddiqui MM, Kongnyuy $M$, et al. Magnetic resonance imaging-transrectal ultrasound guided fusion biopsy to detect progression in patients with existing lesions on active surveillance for low and intermediate risk prostate cancer. J Urol. 2017; 197:640-6.

\section{Correspondence}

Andrea B. Galosi, MD (Corresponding Author)

a.b.galosi@univpm.it

Department of Clinical Sciences, Politecnica delle Marche University

Via Conca 71, 60126 Ancona, Italy

Erika Palagonia, MD

erika.palagonia@gmail.com

Simone Scarcella, MD

simoscarc@gmail.com

Rocco F. Delle Fave, MD

checcoboss90@gmail.com

Angelo Antezza, MD

angelo.antezza@yahoo.it

Lucio Dell'Atti, MD

dellatti@hotmail.com

Division of Urology, School of Medicine, Università Politecnica delle Marche,

Ancona (Italy)

Alessia Cimadamore, $M D$

a.cimadamore@staff.univpm.it

Division of Pathology, School of Medicine, Università Politecnica delle Marche, Ancona (Italy)

Vito Lacetera, MD

vito.lacetera@gmail.com

Division of Urology, Azienda Ospedaliera Marche Nord, Pesaro (Italy) 